\title{
La Teoría de los Sistemas Complejos y la Teoría de los Sistemas Sociales en las controversias de la complejidad
}

\author{
Complex Systems Theory and Social Systems Theory \\ in the controversies of complexity
}

\author{
Gastón Becerra (iD https://orcid.org/0000-0001-9432-8848 \\ Conicet/Universidad de Buenos Aires,Argentina, gaston.becerra@sociales.uba.ar
}

\begin{abstract}
The irruption of complexity in the 1970's and 1980's motivated a certain diagnosis of crisis as regards science and scientific knowledge. Clarifying the scope of this diagnosis is key to understand the meaning acquired by "complexity" in two particular programs: the Theory of Complex Systems (TSC) by Rolando García, and the Theory of Social Systems (TSS) by Niklas Luhmann. To advance on this goal, we propose a counterpoint on 3 particular problems -the scope of explanations and scientific predictions, interdisciplinarity, and the social and transforming nature of knowledgewhich we address with each program's metatheoretical characteristics and epistemological assumptions. We conclude by advocating an interpretation of TSC in which a more moderate position is observed than that in TSS in the controversy of complexity.
\end{abstract}

Key words: complex systems, social systems, complexity, Niklas Luhmann, Rolando García.

Resumen: La irrupción de la complejidad en las décadas de 1970 y 1980 motivó un cierto diagnóstico de crisis de la ciencia y el conocimiento científico. Indagar el alcance de este diagnóstico es central para comprender el sentido que adquiere la complejidad en dos programas particulares: la Teoría de los Sistemas Complejos (TSC) de Rolando García, y la Teoría de los Sistemas Sociales (TSS) de Niklas Luhmann. Para aclarar este problema planteamos un contrapunto en torno a tres problemas particulares -el alcance de las explicaciones y las predicciones científicas, la interdisciplina, y el carácter social y transformador del conocimiento-, que atendemos junto a las características metateóricas y los supuestos epistemológicos de cada programa. Concluimos abogando por una interpretación de la TSC donde

Recepción:

23/02/2019

Aprobación: $18 / 09 / 2019$ se observa una posición más moderada que la de la TSS en la controversia por la complejidad.

Palabras clave: sistemas complejos, sistemas sociales, complejidad, Rolando García, Niklas Luhmann. 


\section{Introducción}

Los estudios en torno a la complejidad y los sistemas complejos comprenden un campo multidisciplinario y poco integrado. Entre las distintas corrientes que reclaman un lugar en el entendimiento de la complejidad podemos destacar dos: por un lado, la tradición sistémico-cibernética que estudia fenómenos de varios dominios con la perspectiva de sistemas y que tiene como principales referentes a Ludwig von Bertalanffy, Ross Ashby, Norbert Weaver, Gregory Bateson y Heinz von Foerster, entre otros; y por otro, a las así llamadas "ciencias de la complejidad", caracterizadas por su enfoque algorítmico para el tratamiento de sistemas adaptativos, dinámicos, no-equilibrados y no-lineales, con referentes como Edward Lorenz, Benoit Mandelbrot o Ilya Prigogine. El sentido de la noción "complejidad" no es unívoco a través de estas corrientes -cuyos desarrollos se han dado con cierta independencia (Phelan, 2001) - ni al interior de ellas -hay más de 40 definiciones aplicables solo a las "ciencias" (Lloyd, 2001)-. Y esto por no mencionar una vasta literatura que identifica la complejidad con una cosmovisión de ideas espirituales, literarias, éticas y políticas.

La irrupción de la complejidad en las décadas de 1970 y 1980 -y aún hoy en sus proyecciones actuales- ha tenido un enorme impacto en la forma en que entendemos el conocimiento científico. En torno a esta irrupción se ha generado una controversia: para algunos autores los estudios sobre la complejidad constituyen una revolución científica en ciernes (por ejemplo, Waldrop, 1992; Wolfram, 2002); para otros es un discurso inflado con tintes anticientíficos (por ejemplo, Horgan, 2015). Esta controversia es el contexto más amplio donde se ubican nuestros análisis (para una defensa de la controversia como marco analítico puede consultarse a Nudler [2009]; para un antecedente de dicho enfoque en el campo de la complejidad, puede consultarse a Rodríguez-Zoya y Rodríguez-Zoya [2014]).

Nuestro objetivo es elucidar el sentido que adquiere la complejidad en dos programas particulares: la Teoría de los Sistemas Complejos de Rolando García (en adelante TSC), y la Teoría de los Sistemas Sociales de Niklas Luhmann (en adelante TSS). Ambos programas tratan con la complejidad y los sistemas complejos de una manera relevante para las ciencias sociales, nutriéndose de los aportes de las dos corrientes mencionadas. Para ello, proponemos ubicar ambos programas en la controversia por la complejidad, haciendo foco en tres problemas particulares: el alcance de las explicaciones y las predicciones de las teorías científicas, la especialización disciplinaria, y el carácter social y político del conocimiento y su capacidad para transformar e intervenir sobre la realidad. 
Este trabajo se organiza de la siguientes manera: en el primer apartado aclaramos brevemente la controversia que, para nosotros, gira en torno a un cierto diagnóstico de crisis que se hace respecto a la ciencia en el nombre de la complejidad, y luego señalamos tres problemas específicos para ordenar el contrapunto; en el segundo apartado incluimos una presentación rápida de la TSC y la TSS - presentaciones más completas de ambos programas han sido desarrolladas en otros trabajos que se citan en contexto-. En el tercer apartado entramos en un contrapunto entre TSC y TSS en relación con cada problema particular; dejamos para la conclusión la síntesis y la evaluación sobre cómo se plantea cada programa frente al diagnóstico de crisis de la ciencia.

\section{La controversia por la complejidad}

Este trabajo parte de la siguiente propuesta para focalizar la controversia que se erige en torno a la complejidad: el quid o centro de dicha controversia es un cierto diagnóstico de crisis de la ciencia y el conocimiento cientifico. El supuesto compartido que habilita esta controversia es que la complejidad introduce cambios y cuestionamientos en algunas características salientes de la ciencia moderna y clásica y del pensamiento científico; lo que está en disputa es el alcance de dichos cuestionamientos. De antemano, podemos suponer dos posiciones:

a) Quienes sostienen que los desarrollos en torno a la complejidad confluyen en una "revolución científica", o que constituyen una "nueva ciencia" con cuestionamientos en múltiples aspectos -fundamentos lógicos, organización social, disposiciones técnicas, método, pretensiones de acción sobre el mundo, etc.-, de modo que su emergencia ha puesto en evidencia una "crisis" de la ciencia.

b) Quienes entienden que los desarrollos sobre la complejidad actualizan a la ciencia "clásica" en algunos aspectos particulares, pero sin llegar a modificar sus rasgos centrales, y que, respecto a los primeros, entienden que en el trasfondo de la prédica "revolucionaria" de la complejidad hay una tendencia "anticientífica".

En vistas a la comparación entre los programas de la TSC y la TSS, aquí proponemos limitar la controversia a tres problemas que alcanzan a la construcción de conocimiento científico y, por esta vía, a las pretensiones científicas que se encuentran en disputa a partir de la complejidad.

El primer problema trata con el alcance de las explicaciones y las predicciones de las teorías científicas. Al respecto, varios autores han sostenido 
que diversas ideas asociadas con la emergencia de la complejidad -como las lógicas autoorganizativas, la no-linealidad, la heterogeneidad constitutiva de los fenómenos complejos, la demostración de la incompletitud, y el reconocimiento de la naturaleza histórico-social y contingente del conocimiento científico-suponen una crítica a algunos rasgos asociados a una ciencia marcada por la física del siglo XVIII -como la tendencia atomista, determinista y causalista-, pero también a las pretensiones explicativas y predictivas de la ciencia desde entonces (Mikulecky, 2007; Najmanovich, 2002).

El segundo de los problemas escogidos trata sobre la tendencia hacia la especialización disciplinaria. La siguiente observación acerca de la recepción de la complejidad en las ciencias sociales es un buen ejemplo de cómo estos desarrollos suelen derivar en interdisciplina:

La complejidad convoca a enlazar y articular la evidente dispersión del conocimiento, con el propósito de aumentar y mejorar la comprensión del mundo natural y social. Para ello, es preciso superar las fronteras disciplinares, realizar una lectura oblicua (no-lineal) de la realidad y articularla con la lógica de la complejidad y la necesidad de expresar cómo se atraviesan las múltiples dimensiones del conocimiento (Aronson, 2013: 17).

El tercer problema que nos interesa abordar remite a la relación cienciasociedad, y alcanza al problema de la intervención sobre la realidad. En los últimos años la ciencia se ha visto interpelada por una sociedad que le presenta problemas y quiere tener voz y voto en sus evaluaciones, en vistas de los potenciales riesgos de sus aplicaciones, de los costos que ella implica, y más aún de su manera como se relaciona con la intervención y la transformación de la realidad. Sería errado decir que la emergencia de la complejidad es la (única) causa de estos reclamos, pero, sin duda, los expresa en tanto la controversia que la envuelve supone el cuestionamiento del sentido y los alcances del conocimiento. Como indica Nowotny (2005), el sentido de "complejidad" se empobrece mucho si no incluye las relaciones intrincadas y las mutuas incertidumbres que se plantean en la coevolución entre ciencia y sociedad.

Los tres problemas mencionados se dirigen al conocimiento científico, sus condiciones de producción y su vinculación con la realidad y la sociedad. Las posiciones de los programas en la controversia se verán fuertemente marcadas por su epistemología, y en tanto los dos programas se inscriben en el amplio campo del constructivismo (Becerra y Castorina, 2018), será necesario consultar sus supuestos en dicho terreno y contrastar sus diferencias para contextualizar y dilucidar sus posiciones respecto a la complejidad. 


\section{Presentación sintética de la TSC y la TSS}

La TSC propuesta por Rolando García es un marco integral de análisis para problemáticas complejas que requieren de un enfoque interdisciplinario (Becerra y Amozurrutia, 2015; García, 2006). La TSC comprende una formulación teórica breve con un lenguaje conceptual y un conjunto de principios generales acerca de la composición, dinámica y evolución de un sistema, lineamientos metodológicos que guían el trabajo interdisciplinar y una fundamentación epistemológica de raíz constructivista.

Para la TSC, la complejidad es un adjetivo que se aplica a un recorte de la realidad, de modo que un objeto de conocimiento es complejo para un sujeto cuando este no cuenta con las capacidades para interactuar con las relaciones de los componentes del sistema. Esto excluye su uso como sustantivo, refiriéndola con independencia de un fenómeno y del sujeto de conocimiento, o habilitando la pregunta: “qué es la complejidad?” (García, 2000: 67).

Por su parte, el concepto de sistema remite a una forma de representar $\mathrm{u}$ organizar fenómenos que presentan componentes de diversa naturaleza material y en las cuales confluyen diferentes procesos en forma interdefinida (García, 2006: 21). En tanto una representación, los sistemas se construyen por parte de un sujeto de conocimiento. Un elemento central de esta construcción es el recorte de algunos elementos de la realidad, relegando otros al entorno. Esta decisión es luego reinsertada en el análisis de la evolución del sistema, siguiendo un modelo de sistema abierto y de estructura disipativa (García, 2006: 60-61).

Una característica paradigmática de la TSC es su interés por construir sistemas en torno a problemáticas que no se limitan al dominio material de una disciplina particular (García, 2006: 33), sino por aquellas que involucran componentes físico-naturales y de las ciencias sociales. Esto vincula la noción mencionada de complejidad con la dificultad de generar un enfoque interdisciplinario. Interdisciplina tiene un significado particular en la TSC: es una metodología que busca lograr un análisis integrado de los procesos que tienen lugar en un sistema complejo y que explican su comportamiento y evolución como totalidad organizada (García, 2006: 88).

La condición que permite este análisis es que el equipo interdisciplinario realice una construcción conjunta del sistema, gracias a compartir un cierto "marco epistémico", entendido como:

[...] el conjunto de preguntas o interrogantes que un investigador se plantea con respecto al dominio de la realidad que se ha propuesto estudiar. Dicho marco epistémico representa cierta concepción del mundo y, en muchas ocasiones, expresa la jerarquía de valores del investigador (García, 2006: 36). 
Como señalamos en otros trabajos (Becerra, 2014b; Becerra y Castorina, 2016a), las preguntas detrás de este enfoque son claramente preteóricas: ¿qué aspecto de la realidad se nos aparece como problemático?; ¿por qué y para qué queremos intervenir y generar conocimientos?

La investigación en sistemas complejos comprende dos momentos: el primero apunta al diagnóstico y consiste en identificar procesos y mecanismos involucrados en la génesis de la problemática; el segundo apunta a la propuesta de un plan de intervención para construir un escenario alternativo. El pasaje de un momento al otro no es lineal. Las transformaciones inducidas en el sistema pueden impactar en áreas poco estudiadas del sistema o su entorno y ocasionar comportamientos no previstos; en cuyo caso la delimitación del sistema debe volver a revisarse, incluso llegando a requerir del aporte de nuevas disciplinas.

La TSS de Niklas Luhmann busca ser una teoría general con la cual delimitar de forma unitaria el campo de estudio de la sociología (Luhmann, 1998). La TSS comprende: un entramado conceptual vasto; lineamientos metodológicos vinculados con la observación de segundo orden; y, al igual que la TSC, una epistemología constructivista. El primero de los (varios) sentidos que encontramos en la TSS en torno a la complejidad supone el tratamiento de la distinción elemento/relación (Luhmann, 1998: 47). Este refiere a una limitación inmanente del sistema en su capacidad de enlace entre elementos, de modo que se ve forzado a reducir complejidad por medio de seleccionar qué relaciones serán actualizadas.

Lejos de significar una simplificación, esta reducción es construcción de una complejidad estructurada bajo la selectividad del sistema, y frente a la complejidad inasible del entorno (Luhmann, 1998: 50). Al igual que en la TSC, este primer sentido trata a la complejidad como un atributo: los sistemas son complejos en vista de esta compulsión a seleccionar. De dicha complejidad, Luhmann (1998: 50) deriva un segundo sentido que se acerca más a un problema cognitivo o reflexivo, al cual refiere con la distinción entre información/indeterminación, que implica la medida de la información que le falta al sistema para realizar sus descripciones del mundo y de sí mismo. En los sistemas que reducen sentido, esta complejidad puede aparecer problematizada como miedo, inseguridad, riesgo, problema de planeación, etcétera.

La reducción de complejidad funciona como el motor de la evolución de los sistemas. La complejidad estructurada por el sistema está sometida a los mismos lineamientos que la han construido, de modo que una vez alcanzado cierto umbral de complejidad, el sistema ya no puede garantizar su unidad y se generarán nuevos sistemas en su interior que reduzcan complejidad con 
distinciones más finas. De la teoría de la complejidad se sigue así una teoría de la diferenciación, fuertemente vinculada con la evolución de sistemas adaptativos.

De acuerdo con Luhmann, en la sociedad se han sucedido diferentes formas de diferenciación, siendo la funcional la que caracteriza a la sociedad moderna. En ella, los sistemas se disponen - para un observador-como formas especializadas (y parciales) en torno al tratamiento de problemas (Becerra, 2013).

En tanto, la comunicación opera de manera fáctica y es empíricamente observable. La TSS se compromete metodológicamente con un trabajo de observación "de segundo orden", entendida como una observación destinada a observar cómo observa otro sistema, cuáles son las distinciones y esquemas que emplea, y cuáles son sus puntos ciegos. En particular, la observación de segundo orden puede ser útil para ver de qué forma distintos sistemas sociales reconstruyen un cierto elemento de su entorno, sometiéndolo a procesamientos diferentes. Luhmann (1998: 72) es explícito en que el sentido de dichas observaciones, en un planteo sistémico-funcionalista, es posibilitar la comparación y abrir lo existente a otras posibilidades.

Este tipo de análisis es característico del sentido que Luhmann otorga a la construcción de teoría: abstraer fenómenos y observaciones construyendo una complejidad propia, que permita establecer comparaciones cada vez más improbables (Luhmann, 1997: 294). A su vez, esta orientación para la teoría avanza un cierto criterio de verdad, fuertemente vinculado con la redundancia y la exigencia de coherencia, siendo estos criterios internos de la operación del sistema ciencia (Becerra, 2014a y 2018).

\section{TSC y TSS en la controversia por la complejidad}

Problema \# 1. El alcance de las explicaciones y las predicciones de las teorias cientificas

Quienes sostienen que los estudios acerca de la complejidad y los sistemas complejos reflejan una crisis en la ciencia, suelen considerar que estos nos enfrentan a un límite en nuestra capacidad para predecir y explicar. Aquí conviene distinguir dos tipos de cuestionamientos que subyacen a este reclamo: los que involucran problemas de explicación -como determinismo, causalidad o emergencia- y su relación con la predicción; y los que involucran problemas epistémicos relativos a la construcción del sistema -como el recorte de sus dominios o la disponibilidad de información acerca de ellos-. 
El principal propósito de la TSC es explicar el funcionamiento y la evolución de un sistema. En este propósito, García (2006) es explícito en que la orientación de las explicaciones de la TSC es causal:

La investigación [en sistemas complejos] consiste en la propuesta de sucesivas modelizaciones hasta llegar a un modelo aceptable, entendiendo por tal un modelo que permite formular explicaciones causales de los fenómenos que son objeto de estudio ... El conjunto de las relaciones allí involucradas constituye la explicación del funcionamiento del sistema (García, 2006: 84 [resaltado nuestro]).

Las "relaciones allí involucradas" a las que refiere la cita son inferencias que vinculan teóricamente los fenómenos recortados. Como hemos señalado en otros trabajos (Becerra y Castorina, 2016a y 2018), el constructivismo al cual subscribe García entiende que un sujeto -aquí la ciencia- construyen conocimiento acercándose de forma asintótica e inacabada a la realidad, tomando nota de las resistencias que la misma le ofrece a sus acciones.

En esta posición, García se encuentra fuertemente vinculado al realismo epistémico que subyace a la obra de Jean Piaget. En consecuencia, los modelos causales a los cuales se recurre en la TSC deben ser coherentes con este supuesto ontológico, y así discurrir por estructuras imbricadas en las que intervienen niveles emergentes de propiedades, fenómenos y comportamientos autónomos -es decir, una causalidad bottom-up constituida sobre relaciones entre elementos-, en balance con las perturbaciones y restricciones de las totalidades sobre las partes - procesos top-down- (García, 2006: 187-190). Que una perturbación desencadene un mecanismo compensador o una completa reestructuración depende tanto de la historia interna de un sistema, como de sus condiciones de contorno.

Ahora bien, la TSC también reconoce que las explicaciones causales para sistemas con estructuras imbricadas difícilmente podrían permitir la predicción de sus futuros estados (García, 2006: 50-51). Pero esta dificultad no implica que el sistema se comporte de forma azarosa, lo cual haría imposible las explicaciones. De hecho, García ha insistido en la necesidad de distinguir predictibilidad y determinismo, especialmente a partir de las enseñanzas sobre el caos determinista de Lorenz y la consideración de sistemas altamente sensibles a condiciones iniciales (García, 2006: 133-134; Inhelder et al., 1981: 144). En este punto los problemas de explicación entroncan con los de la construcción del sistema. O en otras palabras: las limitaciones empíricas en la construcción del sistema se vuelven limitaciones para la explicación. Esta es una situación característica en las pretensiones explicativas de los estudios de la complejidad: 
Porque los sistemas complejos son sistemas abiertos, necesitamos comprender el entorno completo del sistema antes de poder entender el sistema y, por supuesto, el entorno es complejo en sí mismo. No hay manera humana de hacer esto. [...] El problema se ve agravado por el hecho de que lo que queda fuera interactúa con el resto del sistema de una manera no lineal y por lo tanto no podemos predecir cuáles serán los efectos de nuestra reducción de la complejidad (Cilliers, 2005: 258).

Ahora bien, la forma de entender esta limitación es justamente el parteaguas en los estudios de la complejidad. Por ejemplo, a continuación de la cita anterior, Cilliers (2005) nos advierte que somos seres finitos y que nuestra actitud epistémica debería ser suficientemente modesta para permitir la revisión y la crítica, para luego desencadenar en un cierto escepticismo. Por su parte, la incompletitud del conocimiento y la necesidad de su continua revisión están supuestos en el constructivismo de García, tanto en su realismo crítico como en su caracterización del sujeto social cognoscente, y sobre ellos García avanza con una propuesta de trabajo que hace del recorte de los problemas el momento central de la construcción del sistema complejo, y que señala el balance entre la desnaturalización de la realidad y la factibilidad del estudio y los propósitos del equipo como su principal desafío (García, 2006: 95). No obstante, el realismo de su epistemología lo contiene de caer en posiciones posmodernas.

En consecuencia, la TSC afirma que la explicación es posible -si bien estas explicaciones se dirigen a un recorte particular- y que estos problemas pueden ser tratados causalmente -con modelos de causalidad múltiple y circular-. Luego, la predictibilidad es una exigencia difícil, ya que un cierto grado de incertidumbre es inevitable, pero no por ello es una empresa que se debe abandonar, sino que más bien constituye un objetivo fundamental de los estudios de sistemas complejos. El problema es práctico y no metafísico, lo cual justifica que se debe operar sobre la estructuración. Lejos se encuentra esto de constituir una visión devastadora de la ciencia. A lo sumo, la imagen de la ciencia que queda bajo crítica sería una demasiado acotada: aquella que equipara explicación con predicción, y que fundamenta la predicción en una visión determinista de la realidad.

Para la TSS la complejidad se expresa sobre todo como selectividad forzada. En esto no hay una gran diferencia con lo afirmado antes, pero la orientación que toman estos razonamientos en Luhmann son más radicales que los de García.

Coherente con la orientación cibernética, Luhmann (1998) opta por observar al observador observando su entorno, resultando en paradojas. Aquí la TSS bien podría romper estas paradojas con decisiones prácticas, 
pero la concepción antihumanista y antinormativista de su autor (Funes, 2004) funciona como un bloqueo. Al tratar con estas paradojas, su enfoque entra en un círculo de impredecibilidades. Es frente a las problemáticas complejas -como los peligros ecológicos-que este planteo alcanza su tono más característico:

El saber sobre contextos ecológicos crece rápidamente gracias a los conocimientos que las ciencias naturales imponen. Somos conscientes más que cualquier sociedad anterior de la complejidad, y más que en cualquier otra sociedad están a la vista posibilidades de investigación que prometen éxitos. Aunque al mismo tiempo crece — de manera desproporcionada - el no-saber. A eso se acomodan las matemáticas y las técnicas de simulación sólo para venir a reafirmar lo imposible que es pronosticar. La sociedad con su saber en aumento (no a-pesar-de, sino porque su saber aumenta) ya no es capaz de informar sobre las relaciones que se establecen entre cambios societales y cambios en el entorno. Lo que antes se mostraba como un kósmos ordenado, ahora se presenta como ámbito de posibles catástrofes (Luhmann, 2007: 881).

Respecto a los modelos explicativos, estas limitaciones favorecen la adopción de una perspectiva funcional-comparativa en torno a las distintas posibilidades que se abren para la reducción de complejidad desde la óptica de los sistemas. Desde esta perspectiva, las observaciones basadas en relaciones causales aparecen como un intento de asimetrización de la paradoja: son los sistemas autorreferentes los que definen los límites de lo interno/externo, de modo que son ellos quienes estabilizan esta distribución necesaria para cualquier imputación ulterior. No obstante, si bien se habla de explicaciones causales (Luhmann, 1998: 34), la versión del constructivismo en la cual se ubica Luhmann nos obliga a algunas precauciones.

El proyecto epistemológico de Luhmann (1990 y 2006) tiene por centro la radicalización completa del constructivismo, por la vía de considerar a la sociedad como sistema epistémico. Un elemento esencial de dicha radicalización es la negación de toda exteriorización de las construcciones del conocimiento. Luhmann se adhiere a una versión más escéptica del constructivismo que García, remarcando que no hay nada en el entorno que pudiera corresponder con la cognición, dado que esta depende de las distinciones que introduce el sistema con su observación (Becerra y Castorina, 2018). Tal vez sea por este límite que la TSS, a diferencia de la TSC, no da el paso de relaciones a explicaciones causales (en los términos de García).

\section{Problema \# 2. La especialización disciplinaria y los desafios de la interdisciplina}

Otro punto importante en la controversia de la complejidad se da en torno a los límites disciplinarios que organizan la ciencia moderna. Entre los 
reclamos más fuertes de quienes observan en la complejidad una revolución científica, se encuentra la idea de que los estudios sobre sistemas complejos obligan a cruzar -o, en ocasiones, eliminar- dichos límites o fronteras. "Interdisciplina” es el término que recoge este reclamo, uno tan genérico que basta una revisión bibliográfica para ver que esconde una pluralidad de sentidos: en ocasiones se la asocia con una metodología de investigación, a un cuestionamiento de institucionalidad de la ciencia, una filosofía y hasta una ideología (Klein, 1990).

Incluso si nos referimos solo a un vínculo entre aportes teóricos, metodológicos y técnicos de distintas ciencias, observamos tal variedad de formas de interdisciplinariedad, que ya se cuenta con taxonomías (Klein, 2010). Descartamos, entonces, recurrir a una definición como criterio. Más bien, deberemos indagar qué sentidos quedan involucrados cuando la TSC y la TSS hablan de interdisciplina.

Como mencionamos, uno de los componentes centrales de la TSC es una metodología de trabajo interdisciplinaria para equipos de investigación de conformación multidisciplinaria. La distancia entre "multi" e "inter" se puede observar, de acuerdo con García (2006: 32), en el modo de concebir la problemática y el trabajo:

Mientras que en el caso de las investigaciones multidisciplinarias se suelen sumar los aportes que cada investigador realiza desde su disciplina particular en torno a una problemática general que puede ser analizada desde diferentes perspectivas, una investigación interdisciplinaria supone la integración de estos diferentes enfoques para (es decir previa a) la delimitación de una problemática.

En esta definición ya encontramos la idea central de que se necesita una práctica social para generar los instrumentos teóricos, que permitan organizar las interacciones con el sistema. Particularmente, se trata de un enfoque integrado por distintos marcos explicativos, tendiente a la construcción de explicaciones comprensivas sobre un problema con un modelo sintético. El resultado es una interdisciplina que toma la forma de un diálogo (García, 2013). La clave de esta integración es la construcción conjunta del objeto de estudio -el recorte del sistema complejo, propiamente dicho-. La TSC señala como principal condición para esta construcción conjunta que el sujeto cognoscente -equipo de investigación- comparta un diagnóstico y una pregunta rectora. En la cita anterior se habla de un enfoque común. Para aclarar lo que aquí está en juego se debe volver al terreno de la epistemología.

En el constructivismo que elabora García, con base en su colaboración con Piaget, se destaca una noción particular: la del marco epistémico. Si bien la misma ha adquirido distintos significados en varios momentos de su obra 
(Becerra y Castorina, 2015), entendemos que en el núcleo de su definición se encuentra el condicionamiento de lo social sobre lo cognitivo (Becerra y Castorina, 2016b). De allí que la TSC la define como una "normatividad extra-disciplinaria de contenido social” (García, 2006: 106), que es incorporada a las teorizaciones por la vía de la práctica estructurante.

Más específicamente, el marco epistémico se expresa en el recorte de la problemática, en vista de ciertos intereses sociales y políticos. Luego, se pone en movimiento una marcha dialéctica de integraciones y diferenciaciones de conocimiento de especialistas que constituyen el centro de la actividad estructurante y que llevan a la construcción de un modelo.

Por su parte, los objetivos programáticos de la TSS se relacionan en un sentido distinto con la interdisciplina. En la TSS, la especialización disciplinaria es el resultado de la diferenciación interna de la ciencia, en vista de su forma de construir una mayor complejidad, y como forma de superar los rendimientos de una ciencia ordenada jerárquicamente (Luhmann, 1997: 327). La interdisciplina, en esta visión, es la forma en que se evidencia la interdependencia de dichos sistemas especializados (Luhmann, 1997: 327329). Luhmann caracteriza tres situaciones:

1. El contacto casual u ocasional entre programas paralelos de las ciencias (teorías y métodos de distintas disciplinas), por el cual una disciplina incorpora un producto de otra con efectos inesperados en la ciencia que lo incorpora.

2. La cooperación entre disciplinas en torno a un problema y en el marco de un proyecto de investigación que delimita un cierto tiempo de trabajo y lo regula a través de objetivos -aquí podríamos ubicar la interdisciplina de la TSC-.

3. Un esfuerzo de trascender los límites disciplinarios, proponiendo distinciones abstractas sobre las cuales se puedan fundar nuevos paradigmas.

En tanto, el interés que motiva a Luhmann es el de revitalizar la teorización sociológica, la colaboración interdisciplinaria a la que apunta no excede el diálogo teórico. Si bien Luhmann explícitamente se reconoce dentro de la primera situación de interdisciplina -en especial por la incorporación de la autopoiesis a la sociología-, es claro que tiende al tercero - con el desarrollo de la cibernética y la teoría de sistemas-.

Se trataría de tender puentes conceptuales al poner el foco en una teorización particular (sistemas), el planteo de analogías entre los dominios y su traducción a un lenguaje más riguroso. En cualquier caso, Luhmann no aporta ningún método, receta, guía o recomendación para este desarrollo. 
Se podría conjeturar que esta falta de lineamientos se debe a los esfuerzos de Luhmann por librar a la ciencia de todo tipo de normativismos, priorizando la "libertad inventiva" de la teoría, por utilizar la expresión - tal vez irónicade Zolo (1995).

Para la TSS, una teoría novedosa es una propuesta comunicativa en la evolución de la ciencia, un pre-adaptative advance a la espera de condiciones propicias (metodológicas, técnicas, educativas, etc.) para ser seleccionada y replicada. Incluso, frente a la crítica usual de que la TSS es incompatible con la ciencia empírica, Luhmann (1992: 1439) sugiere que la culpa está en la falta de métodos y técnicas más avanzadas para las ciencias sociales. Esto plantea la pregunta de si acaso las relaciones con las ciencias de la complejidad podrían dar este paso, siendo este un problema derivado para la comparación de la TSC y la TSS.

Como mencionamos en la introducción, el enfoque sistémico-cibernético y las ciencias de la complejidad no comparten una historia común, surgiendo las primeras al menos tres décadas antes que las últimas y teniendo un enfoque mucho más general y abarcativo. Desde entonces la orientación de ambas ha tomado cursos divergentes: la tradición sistémico-cibernética ha profundizado la reflexión epistemológica -en la cual el constructivismo juega un rol central-, impulsado y problematizado la práctica interdisciplinaria, se han abierto a incorporar técnicas y enfoques (cualitativos) de las ciencias sociales, todos estos aspectos muy poco explorados en las "ciencias"; en contrapartida, estas últimas han desarrollado una buena cantidad de modelos formales y han avanzado el estado del arte de técnicas y herramientas computacionales (Castellani y Hafferty, 2009; Maldonado, 2012; Reynoso, 2006).

En el plano técnico-metodológico, a la hora de referir a la relación entre la TSC y las ciencias de la complejidad, la escasa literatura se ha hecho eco de un pasaje que conviene citar:

En décadas recientes ha surgido una vasta literatura acerca de lo que se suele llamar, a mi juicio erróneamente, 'las ciencias de la complejidad'. Como es sabido, el desarrollo explosivo de esta literatura se debe, en gran medida, a la introducción de computadoras de gran capacidad, extraordinariamente rápidas, que permiten atacar problemas que estaban antes fuera del alcance de los métodos matemáticos. La variedad de problemas que fueron abordados, y la cantidad de resultados espectaculares obtenidos, han permitido extender enormemente la comprensión de los procesos no-lineales, pero ello ha llevado también, lamentablemente, a lo que me he permitido llamar extrapolaciones matemáticas ilegítimas y falacias correlacionadas. Prevalece en la ciencia actual un cierto imperialismo de las computadoras que hace aparecer como no-científico todo estudio de procesos no "modelables" a través de un sistema de ecuaciones diferenciales no-lineales (o de otras modelizaciones más sofisticadas) (García, 2006: 75). 
En nuestra lectura, este pasaje debe ser interpretado como una advertencia a no desestimar la problemática epistémica que aquí ubicamos en el corazón de la TSC, lo que está lejos de negar el uso de modelos y técnicas computacionales en el tratamiento de problemas complejos. De hecho, adjudicarle al autor de la TSC una posición en contra de estas herramientas sería incoherente considerando que se trata de quien no solo impulsó la radicación de la primera computadora científica de Latinoamérica, sino también de quien trabajó con los primeros modelos predictivos en problemática climática, además de la explícita evaluación positiva acerca de su utilidad en ciencias sociales como instrumentos prospectivos de situaciones poco exploradas (García, 2006: 134).

En todo caso, el problema no es la técnica en sí ni la posibilidad de cuantificación efectiva de ciertos fenómenos, lo cual, a lo sumo, sería una limitación metodológica de cada época. Lo que se critica en estas "importaciones” es la incorporación inadvertida de supuestos filosóficos -como el determinismo o el reduccionismo- y posiciones meta-teóricas que inciden en las técnicas -como el reduccionismo de algunas simulaciones-, incongruentes con la visión que defiende la epistemología constructivista en la cual se sustenta la TSC.

Por su parte, la TSS prácticamente no incorpora elementos técnicos o metodológicos de las ciencias de la complejidad, tal vez por la falta de lineamientos metodológicos más profundos. Desarrollos recientes, como los que incluyen técnicas de simulación y cálculos de entropía en la comunicación y de emergencia de atractores, podrían revertir esta situación, pero por el momento su aplicación no va más allá de ilustrar aspectos parciales de la teoría, y el estado muy embrionario de estos esfuerzos no nos permite suponer que tengan la capacidad de dotar a la TSS con un enfoque que le permita repensar su tratamiento de la complejidad.

Dado el lugar hegemónico que la TSS aún hoy tiene como exponente de la complejidad en las ciencias, esta particular relación con las "ciencias de la complejidad" nos deja frente a dos lecturas enfrentadas: en una lectura optimista, Luhmann podría ser un dinamizador del interés por la complejidad; o bien podría ser un distractor de los desarrollos más novedosos de las ciencias de la complejidad. Aquí creemos que la TSS probablemente sea ambas al mismo tiempo: un dinamizador del interés por una complejidad en sentido amplio - que alcanza a una reflexión epistemológica y que incluye también un cuestionamiento de la complejidad como categoría social y epistémica-; y un distractor de la incorporación de técnicas y modelos. 
Problema \# 3. La relación conocimiento-sociedad y la intervención sobre la realidad

El último problema que nos interesa tratar de la controversia por la complejidad refiere al propósito que se persigue en la construcción de conocimiento científico, algo que ubicamos en la relación ciencia-sociedad.

El objetivo de la TSC respecto a la complejidad es problematizar las herramientas del conocimiento por medio de una reflexión sobre el sujeto epistémico, sus estructuras y acciones, para con ellas transformar la realidad. En esta relación conocimiento-sociedad-realidad reaparecen dos elementos centrales del constructivismo en el que se asienta la TSC.

Por un lado, como mencionamos sobre la noción de marco epistémico, el recorte y el señalamiento de las problemáticas a las que se orienta la construcción del sistema complejo en el juego interdisciplinario se encuentran condicionadas por una concepción del mundo y de ciertos valores sociales y políticos, que ponen en juego un cierto estado ideal del sistema. Luego, las modelizaciones tienden a la búsqueda de soluciones: acciones deliberadas (estrategias, tácticas y operaciones) sobre los mecanismos causales (procesos), que se han identificado en la etapa de diagnóstico. Aquí se observa por qué es fundamental para la TSC una cierta compatibilidad con el realismo en torno a la noción de sistemas.

Por otro lado, si bien esta consideración de lo social en la construcción epistémica de los sistemas es "de avanzada" en el contexto de los debates acerca de la interdisciplina (Boix-mansilla, 2006), si consideramos al sujeto de conocimiento de la epistemología de García, nos encontramos con una tensión: la interdisciplina que promueve la TSC es "de especialistas". Esto parece no tomar en cuenta los saberes, sentidos y valoraciones de los actores sociales involucrados en las problemáticas.

De hecho, en la TSC, la participación pública queda limitada al señalamiento de las problemáticas (García, 1990: 570). Estas limitaciones dejan a la TSC a tiro de la crítica de cientificismo. Se podría conjeturar que este punto ciego tal vez no se deba al posicionamiento político de su autor, sino más bien a su condicionamiento disciplinario, ya que García era un físico ajeno a las clases interactivas de las ciencias sociales; sin embargo, también debería evaluarse cuánto peso tiene en este límite que la conceptualización sistémica de la TSC se acerque más a una primera cibernética, antes que a la segunda de los giros autorreferenciales (Becerra, 2016).

En cualquier caso, los trabajos que han revisado esta parte de la TSC (por ejemplo, Amozurrutia, 2012) sugieren incluir -en el momento epistémico- 
técnicas cualitativas que promuevan el análisis conjunto con los actores, y luego -en el momento prospectivo- la discusión de los diferentes escenarios en talleres y grupos de trabajo con las comunidades locales para asegurar que las acciones y políticas cuenten con la legitimidad suficiente como para poder ser implementadas.

Por su parte, la noción de "problema" que queda involucrada en la TSS - particularmente en su reflexión metodológica funcionalista- no es equivalente a la categoría de "problemáticas": mientras las primeras son dependientes de la posición de observación de cada sistema, las segundas son preteóricas y su asiento es el analista. Cuando Luhmann hace el cambio de sujeto de conocimiento del individuo hacia una sociedad diferenciada, fragmenta la base del marco epistémico que podría constituir una problemática, para reinsertarla en cada sistema. Aquí, de vuelta, la relación conocimientociencia-sociedad nos remite a dos aspectos característicos de la epistemología de Luhmann.

En primer lugar, las consecuencias de esta fragmentación son grandes: "Los problemas ecológicos no pueden ser abordados como unidad que se pudiera resolver unánimemente en la sociedad, sino que esta unidad se descompone en las perspectivas distintas de los sistemas de funciones" (Luhmann, 1997: 149). Fuchs y Hofkirchner (2009: 114) han visto en esta posición el impacto del constructivismo radical que tiende a poner en duda la existencia de problemas reales para reemplazarlos por problemas construidos en la comunicación.

La diferenciación supone además que una vez reconocido el problema, los sistemas generan una complejidad propia al respecto, de modo que se duplican los espacios de resonancia (en la sociedad) en los que una modificación de la realidad podría desencadenar nuevos desequilibrios (Luhmann, 1989: 118-120). Esto redunda en una visión desalentadora de la intervención en la TSS, en vistas de la complejidad. El tono del autor merece ser resaltado:

No debe causar sorpresa que estos análisis no conduzcan al ofrecimiento de consejos que vayan de acuerdo con el formato de los problemas ecológicos... [U]nos cambios relativamente endebles pueden provocar, en otros sistemas, cambios sobreproporcionados. [...] En vistas de la típica diferenciación funcional, por tanto, de cara a una alta complejidad que se refuerza y se disminuye a sí misma, y que las causalidades no se pueden controlar centralmente, un tal desarrollo catastrófico es, a la larga, probable (Luhmann, 2012: 91).

El lugar que esto deja para el sistema social Ciencia frente a las problemáticas complejas sigue la metáfora epistemológica fundante del constructivismo de Luhmann: (solo) observar. Más específicamente, observar la 
observación de los demás sistemas y aportar a su reflexión acerca de los riesgos y las latencias. En palabras de Luhmann (1989: 131), se trata más de la “[...] expansión las perspectivas sobre los problemas, antes que de su eliminación”. Sin embargo, uno podría pensar que no es tanto la idea de intervención en sí la cual se rechaza, sino una simplista o jerárquica que espera que la ciencia dicte cursos de acción para los demás sistemas sociales.

De hecho, en Ecological communication, Luhmann deja abierta la posibilidad de una intervención no-jerárquica - con consideración de la resonancia propia de distintos sistemas, sus procesos locales, sus escalas de tiempo y retroalimentaciones- sobre problemas complejos, algo que ha tenido bastante eco en la literatura (Buchinger, 2006; Mascareño, 2011; Wan, 2011).

No obstante, y en segundo lugar, esta posibilidad parece ir a contramano de la orientación antihumanista y antinormativista de la TSS. Respecto al antihumanismo, se debe evaluar de qué manera los valores sociales y las acciones de los individuos (por ejemplo, equipo que trata con la problemática) pueden condicionar a los desarrollos de la ciencia. En consideraciones acerca de la ciencia, Luhmann (1997: 419-420) fue explícito en negar esta posibilidad, con un planteamiento que vincula la autopoiesis con la objetividad, entendida en el sentido más limitado de ausencia de valores. Tal vez por ello algunos autores que piensan la intervención sistémica llegan al punto de promover una revisión crítica de la autopoiesis (Fuchs y Hofkirchner, 2009).

Respecto al antinormativismo, se debe señalar que el sentido que Luhmann le confiere es el de evitar la introducción de asimetrías que condicionen la observación con vista al rendimiento teórico. La respuesta, tal vez, aquí es más simple: a varias décadas de la formulación de la teoría, se puede afirmar que esta ha rendido frutos suficientes como para que se pueda suspender dicha exigencia sin limitar el alcance de sus observaciones.

\section{Conclusiones: el diagnóstico de crisis de la ciencia en nombre de la complejidad}

Comenzamos el trabajo proponiendo una lectura de la controversia por la complejidad que ubicaba en el centro de la disputa a un cierto diagnóstico de crisis de la ciencia y el conocimiento científico en vista de la emergencia de la complejidad. Que los desarrollos acerca de la complejidad introducen cambios, innovaciones y cuestionamientos en algunos aspectos de la ciencia es algo trivial. La controversia se despliega en torno al pretendido alcance y la naturaleza de estos cambios: ¿̨los desarrollos de la complejidad nos enfrentan a una nueva imagen de la ciencia, cuyas pretensiones y prácticas difieren de 
la ciencia "clásica"?, ¿los desarrollos de la complejidad actualizan a la ciencia en algunos aspectos sin modificar sus rasgos centrales y sus orientaciones más características?

La posición de la TSC respecto al problema \# 1 que hemos analizado nos permite observar que este programa se encuentra lejos de denunciar una crisis devastadora en la ciencia y el pensamiento científico a partir de las consideraciones de la complejidad. Los principales desafíos en la explicación y la predicción de sistemas complejos no llevan a la exigencia de nuevos marcos de entendimiento, ni a criticar las actuales herramientas del pensamiento científico ni tampoco a renunciar a pretensiones icónicas de la ciencia, como avanzar nuestro conocimiento de la realidad.

Sí se critica, no obstante, una visión simplista de la ciencia que sostiene tales objetivos en supuestos como el atomismo o la linealidad. Y sí se ofrecen algunas advertencias: acerca de la dificultad de generalizar conclusiones por fuera de las consideraciones locales de cada sistema, o acerca de la necesidad de aceptar que no toda explicación será plausible de ser expresada en términos formales. Estas advertencias parecen estar dirigidas solo a quienes pretenden evaluar los estudios de la complejidad bajo los criterios de las ciencias físicas y lógico-matemáticas.

A lo sumo, lo que la TSC propone como algo crítico -en el sentido de una innovación necesaria y urgente- es la necesidad de recurrir a la interdisciplina para tratar con ciertos problemas. En la discusión de los problemas \# 2 y \# 3 observamos las condiciones y los límites de esta propuesta. Es claro que la TSC no niega que haya otros problemas para los cuales una ciencia disciplinaria se haya probado suficientemente exitosa. A la vez, se afirma que los conocimientos de especialistas serán los principales aportes a la investigación interdisciplinaria. Pero para ello es necesario asegurar la integración de los conocimientos disciplinarios, para lo cual la TSC propone una metodología sustentada en su epistemología constructivista. Entre los distintos aportes que la epistemología hace a esta propuesta metodológica, se encuentra el advertir la importancia de una actividad estructurante conjunta en el momento epistémico de la investigación, y en evidenciar el rol constituyente del marco epistémico -y por esta vía de los valores sociales- del equipo multidisciplinario.

En resumen, la TSC aparece como un claro exponente de una posición moderada en la controversia, que defiende los rasgos y criterios de la ciencia moderna, y que entiende que los desarrollos de la complejidad aportan caminos para actualizarla y renovarla con miras a potenciar su capacidad para enfrentar desafíos actuales. 
Por su parte, la TSS introduce un diagnóstico de crisis más fuerte, aunque a la vez, más acotado: lo necesario y urgente para Luhmann es fortalecer la capacidad teórica de la sociología, y su propuesta para ello consiste en ofrecer una teoría universal y abierta al diálogo entre disciplinas. A nuestro entender, esta crisis está vinculada mayormente al sentido epistémico de la complejidad: si la sociología necesita incrementar su complejidad teórica no es en vista de las propiedades de la realidad -lo que sería una justificación ontológica contraria a su escepticismo-, sino en comparación a los logros internos de otras disciplinas.

Esta definición del problema, junto a su posición epistemológica y al camino que toman sus explicaciones, introducen elementos que algunos seguidores de este programa suelen entender como reclamos en contra de la ciencia tradicional. Uno de estos reclamos es el de "liberar a la teoría" de sus rendimientos empíricos y su aplicabilidad práctica como principales criterios de evaluación, para permitirle dedicarse a la observación (por ejemplo, Granja-Castro, 2001).

El carácter autorreferencial y cerrado de la ciencia, junto con la función y el sentido de la construcción teórica que Luhmann propone, dan sustento a dicho reclamo. Otro reclamo puede ser la aceptación de paradojas, como herramientas lógicas válidas y coherentes con los mandatos cibernéticos (por ejemplo, Castro-Sáez, 2011). Las paradojas se justifican en la epistemología de Luhmann por la preeminencia de la diferencia en el tratamiento de las dualidades, y la TSS nos enfrenta a ellas cuando adopta el camino metodológico de la observación de segundo orden, y cuando apunta a dar cuenta de la autorreferencia. Pero se debe notar que estas paradojas se circunscriben a la reflexión del sistema, especialmente al tratamiento de problemas epistemológicos.

Si desde aquí se hace algún reclamo crítico contra la ciencia, este no parece ir más allá de la denuncia acerca de que ciertas construcciones lógicas son intentos por esconder una dualidad. Al menos en el tratamiento de este problema, no parece haber en la TSS el diagnóstico de crisis tan radical que observan algunos de sus lectores, que han llegado a pregonar un nuevo paradigma de pensamiento. El posicionamiento de la TSS en el problema \# 2 que hemos tratado es coherente con la posición adoptada en el problema \# 1: la interdisciplina aporta a una teorización más compleja, dentro de los límites ya señalados.

El diagnóstico de crisis se vuelve más radical recién en el problema \# 3, donde los supuestos epistemológicos de Luhmann y su visión de la ciencia parecen bloquear toda práctica de transformación que pudiera hacer más 
evidente el carácter social y político del conocimiento. Pero, como señalamos, esto puede discutirse, al costo de introducir revisiones en la teorización de Luhmann, y más importante aún, de explicitar y cuestionar algunos de los supuestos epistemológicos y metateórico que dan identidad a su programa. Hasta entonces, la posición de la TSS en la controversia es más radical que la de la TSC, y da lugar a lecturas que permiten sostener reclamos en contra de algunos rasgos tradicionales de la ciencia, que a la vista de sus críticos pueden parecer ataques posmodernos.

\section{Referencias}

Amozurrutia, José Antonio (2012), Complejidad y sistemas sociales: un modelo adaptativo para la investigación interdisciplinaria, México: Universidad Nacional Autónoma de México, Centro de Investigaciones Interdisciplinarias en Ciencias y Humanidades.

Aronson, Perla (2013), La teoría de la complejidad y la complejidad de la teoría, Argentina: Ciccus.

Becerra, Gastón (2013), "Presentación sintética de la teorías de los sistemas sociales autopoiéticos y la teoría de los sistemas sociopoiéticos funcionales de Niklas Luhmann", en Intersticios. Revista Sociológica de Pensamiento Crítico, núm. 7, vol. 2, España: Universidad Complutense de Madrid.

Becerra, Gastón (2014a), "Ciencia y conocimiento en la teoría de los sistemas sociales de Niklas Luhmann”, en Sociología y Tecnociencia. Revista Digital de Sociologia del Sistema Tecnocientifico, núm. 4, vol. 2, España: Universidad de Valladolid.

Becerra, Gastón (2014b), "Interdisciplina y sistemas complejos. Un enfoque para abordar problemáticas sociales complejas", en $\{$ PSOCIAL $\}$ Revista de Investigación en Psicología Social, núm. 1, vol. 1, Argentina: Universidad de Buenos Aires.

Becerra, Gastón (2016), "Sociocibernética: tensiones entre sistemas complejos, sistemas sociales y ciencias de la complejidad", en Athenea Digital, núm. 16, vol. 3, España: Universitat Autònoma de Barcelona.

Becerra, Gastón (2018), "La epistemología constructivista de Luhmann. Objetivos programáticos, contextos de discusión y supuestos filosóficos”, en Sociológica, núm. 33, vol. 95, México: Universidad Autónoma Metropolitana.

Becerra, Gastón y Amozurrutia, José Antonio (2015), "Rolando García’s Complex Systems Theory and its relevance to sociocybernetics", en Journal of Sociocybernetics, núm. 13, vol. 1, España: Research Commitee on Sociocybernetics, International Sociological Association.

Becerra, Gastón y Castorina, José Antonio (2015), "El condicionamiento del marco epistémico en distintos tipos de análisis constructivista”, en Ahumada, José et al. [eds.], Filosofia e historia de la ciencia en el Cono Sur. Selección de trabajos del IX Encuentro y las XXV Jornadas de Epistemología e Historia de la Ciencia, Argentina: Universidad Nacional de Córdoba.

Becerra, Gastón y Castorina, José Antonio (2016a), "Acerca de la noción de 'marco epistémico' del constructivismo. Una comparación con la noción de 'paradigma' de Kuhn”, en Revista Iberoamericana de Ciencia, Tecnologia y Sociedad, núm. 11, vol. 31, Argentina: Centro REDES / Conicet. 
Becerra, Gastón y Castorina, José Antonio (2016b), "Una mirada social y política de la ciencia en la epistemología constructivista de Rolando García”, en Ciencia, Docencia y Tecnología, núm. 27, vol. 52, Argentina: Universidad Nacional de Entre Ríos.

Becerra, Gastón y Castorina, José Antonio (2018), “Towards a Dialogue Among Constructivist Research Programs", en Constructivist Foundations, núm. 13, vol. 2, Bélgica: Vrije Universiteit.

Boix-mansilla, Verónica (2006), "Interdisciplinary work at the frontier: An empirical examination of expert interdisciplinary epistemologies", en Issues in integrative Studies, núm. 31, vol. 24, Estados Unidos: Oakland University.

Buchinger, Eva (2006), "The Sociological Concept of Autopoiesis: Biological and Philosophical Basics and Governance Relevance”, en Kybernetes, núm. 35, vol. 3-4. DOI: 10.1108/03684920610653674 Disponible en: https://www.emerald.com/insight/ content/doi/10.1108/03684920610653674/full/html [17/ de octubre de 2014].

Castellani, Brian y Hafferty, Frederic (2009), Sociology and Complexity Science. A New Field of Enquiry, Alemania: Springer Berlin Heidelberg. DOI: 10.1007/978-3-540-88462-0

Castro-Sáez, Bernardo (2011), “Aportes de Niklas Luhmann a la teoría de la complejidad", en Polis. Revista Latinoamericana, núm. 29, Chile: Universidad de Los Lagos.

Cilliers, Paul (2005), “Complexity, Deconstruction and Relativism”, en Theory, Culture \& Society, núm. 22, vol. 5. DOI: 10.1177/0263276405058052 Disponible en: https:// journals.sagepub.com/doi/10.1177/0263276405058052 [02 de abril de 2007].

Fuchs, Christian y Hofkirchner, Wolfgang (2009), "Autopoiesis and Critical Social Systems Theory”, en Magalhães, Rodrigo y Sánchez, Ronald [eds.], Autopoiesis in Organization Theory and Practice, Reino Unido: Emerald.

Funes, Ernesto (2004), “Acción y sistema en perspectiva: del humanismo al luhmannianismo en la moderna teoría social”, en De Ipola, Emilio [ed.], El eterno retorno. Acción y sistema en la teoría social contemporánea, Argentina: Biblos.

García, Rolando (1990), "Dialéctica de la integración en la investigación interdisciplinaria”, en Cuartas Jornadas de Atención Primaria de la Salud y Primeras de Medicina Social, Argentina: Comisión Argentina de Residentes del Equipo de Salud.

García, Rolando (2000), El conocimiento en construcción: de las formulaciones de Jean Piaget a la teoria de sistemas complejos, España: Gedisa.

García, Rolando (2006), Sistemas complejos. conceptos, método y fundamentación epistemológica de la investigación interdisciplinaria, España: Gedisa.

García, Rolando (2013), "Investigación interdisciplinaria de sistemas complejos: lecciones del cambio climático”, en INTERdisciplina, núm. 1, vol. 1. DOI: 10.22201/ ceiich.24485705e.2013.1.46545 Disponible en: http://www.revistas.unam.mx/ index.php/inter/article/view/46545 [04 de agosto de 2014].

Granja-Castro, Josefina (2001), "El pensar sistémico. Lógicas de razonamiento y horizonte de inteligibilidad en Niklas Luhmann”, en Metapolítica, núm. 5, vol. 20, México: Centro de Estudios de Política Comparada.

Horgan, John (2015), The End of Science, Estados Unidos: Perseus.

Inhelder, Barbel et al. (1981), Jean Piaget. Epistemologia Genética y Equilibración, España: Fundamentos.

Klein, Julie (1990), Interdisciplinarity: History, Theory and Practice, Estados Unidos: Wayne State University Press.

Klein, Julie (2010), "A taxonomy of interdisciplinarity”, en Frodeman, Robert et al. [eds.], The Oxford Handbook of Interdisciplinarity, Estados Unidos: Oxford University Press. 
Convergencia Revista de Ciencias Sociales, vol.27, 2020, Universidad Autónoma del Estado de México

Lloyd, Seth (2001), "Measures of complexity: a nonexhaustive list", en Control Systems, núm. 21, vol. 4, Estados Unidos: IEEE Control Systems Society.

Luhmann, Niklas (1989), Ecological Communication, Estados Unidos: University of Chicago Press.

Luhmann, Niklas (1990), "The cognitive program of constructivism and a reality that remains unknown”, en Krohn Walter et al. [eds.], Selforganization. Portrait of a Scientific Revolution, Países Bajos: Springer.

Luhmann, Niklas (1992), "Operational Clousure and Structural Coupling: The Differentiation of the Legal System”, en Cardozo Law Review, núm. 13, Estados Unidos: De Novo.

Luhmann, Niklas (1997), La ciencia de la sociedad, México: Universidad Iberoamericana, Instituto Tecnológico y de Estudios Superiores de Occidente, Anthropos.

Luhmann, Niklas (1998), Sistemas sociales. Lineamientos para una teoría general, España: Anthropos y Universidad Iberoamericana.

Luhmann, Niklas (2006), "Cognition as construction”, en Moeller, Hans Georg. [ed.], Lubmann Explained: From Souls to Systems, Estados Unidos: Open Court.

Luhmann, Niklas (2007), La sociedad de la sociedad, Mexico: Herder y Universidad Iberoamericana.

Luhmann, Niklas (2012), “¿Puede la sociedad moderna evitar los peligros ecológicos?”, en Argumentos, núm. 25, vol. 69, México: Universidad Autónoma Metropolitana.

Maldonado, Carlos (2012), Derivas de la complejidad, Colombia: Universidad del Rosario.

Mascareño, Aldo (2011), "Sociología de la intervención: orientación sistémica contextual", en Revista Mad, núm. 25, Chile: Universidad de Chile.

Mikulecky, Donald (2007), "Causality and Complexity: The Myth of Objectivity in Science", en Chemistry and Biodiversity, núm. 4, vol. 10. DOI: 10.1002/cbdv.200790202 Disponible en: https://onlinelibrary.wiley.com/doi/abs/10.1002/cbdv.200790202 [28 de abril de 2017].

Najmanovich, Denise (2002), "From Paradigms to Figures of Thought", en Emergence, núm. 4, vol. 1-2, Estados Unidos: Emergent Publications.

Nowotny, Helga (2005), “The Increase of Complexity and its Reduction: Emergent Interfaces between the Natural Sciences, Humanities and Social Sciences", en Theory, Culture \& Society, núm. 22, vol. 5. DOI: 10.1177/0263276405057189 Disponible en: https:// journals.sagepub.com/doi/10.1177/0263276405057189 [19 de junio de 2014].

Nudler, Oscar (2009), Espacios controversiales. Hacia un modelo de cambio filosófico y cientifico, Argentina: Miño y Dávila.

Phelan, Steven (2001), "What is complexity science, really?”, en Emergence, núm. 3, vol. 1. DOI: 10.1207/S15327000EM0301_08 Disponible en: https://www.tandfonline. com/doi/abs/10.1207/S15327000EM0301_08 [28 de abril de 2017].

Reynoso, Carlos (2006), Complejidad y el caos : Una exploración antropológica, Argentina: SB.

Rodríguez-Zoya, Leonardo y Rodríguez-Zoya, Paula (2014), "El espacio controversial de los sistemas complejos”, en Estud.filos, vol. 50, Colombia: Instituto de Filosofía de la Universidad de Antioquia.

Waldrop, Mitchel (1992), Complexity. The Emerging Science at the Edge of Order and Chaos, Estados Unidos: Simon \& Schuster.

Wan, Poe (2011), "Systems Theory: Irredeemably Holistic and Antithetical to Planning?", en Critical Sociology, núm. 37, vol. 3. DOI: 10.1177/0896920510380067 Disponible 
en: https://journals.sagepub.com/doi/abs/10.1177/0896920510380067 [09 de marzo de 2014].

Wolfram, Stephen, (2002), A New Kind of Science, Estados Unidos: Wolfram Media.

Zolo, Danilo (1995), "Autopoiesis: crítica de un paradigma posmoderno", en Zona Abierta, núm. 70-71, España: Fundación Pablo Iglesias.

Gastón Damián Becerra. Doctor en Filosofía, Universidad de Buenos Aires. Becario posdoctoral en Conicet y docente e investigador en Universidad de Buenos Aires y Universidad Abierta Interamericana. Líneas de investigación: epistemología, filosofía de la ciencia, complejidad, big data. Publicaciones recientes: Becerra, Gastón Damián (2018), "La epistemología constructivista de Luhmann. Objetivos programáticos, contextos de discusión y supuestos filosóficos”, en Sociológica, núm. 33, vol. 95, México: Universidad Autónoma Metropolitana. Becerra, Gastón Damián (2018), “Interpelaciones entre el Big data y la Teoría de los sistemas sociales. Propuestas para un programa de investigación”, en Hipertextos, núm. 6, vol. 9, Argentina: Centro CTS, Universidad Maimónides. Becerra, Gastón Damián y Castorina, José Antonio (2018), “Towards a Dialogue Among Constructivist Research Programs”, en Constructivist Foundations, núm. 13, vol. 2, Bélgica: Vrije Universiteit. 